\title{
One of Two Major Paralogs of AVR-Pita1 Is Functional in Japanese Rice Blast Isolates
}

\author{
Mami Takahashi, Taketo Ashizawa, Kazuyuki Hirayae, Jouji Moriwaki, Teruo Sone, Ryoichi Sonoda, \\ Masako Tsujimoto Noguchi, Susumu Nagashima, Kouji Ishikawa, and Michiyoshi Arai
}

First, second, third, and fourth authors: National Agriculture and Food Organization, National Agricultural Research Center, Hokuriku Research Center, 1-2-1 Inada, Joetsu, Niigata, 943-0193, Japan; fifth author: Research Faculty of Agriculture, Hokkaido University, Sapporo, Hokkaido, 060-8589, Japan; sixth author: National Agriculture and Food Organization, National Institute of Vegetable and Tea Science, Shimada, Shizuoka, 428-8501, Japan; seventh author: National Institute for Agro-Environmental Sciences, Kannondai, Tsukuba, Ibaraki, 305-8604, Japan; eighth author: Shimane Agricultural Technology Center, Ashiwata 2440, Izumo, Shimane, 693-0035, Japan; ninth author: Niigata Agricultural Research Institute, 857 Nagakura, Nagaoka, Niigata, 940-0826, Japan; and tenth author: National Agriculture and Food Organization, National Agricultural Research Center for Kyushu Okinawa Region, 2421 Suya, Koshi, Kumamoto 861-1192, Japan.

Current address of third author: National Agriculture and Food Organization, National Agricultural Research Center for Kyushu Okinawa Region, 2421 Suya, Koshi, Kumamoto 861-1192, Japan; current address of tenth author: National Agriculture and Food Organization, National Agricultural Research Center, Hokuriku Research Center, 1-2-1 Inada, Joetsu Niigata, 943-0193, Japan.

Accepted for publication 26 February 2010.

\begin{abstract}
Takahashi, M., Ashizawa, T., Hirayae, K., Moriwaki, J., Sone, T., Sonoda, R., Noguchi, M. T., Nagashima, S., Ishikawa, K., and Arai, M. 2010. One of two major paralogs of AVR-Pital is functional in Japanese rice blast isolates. Phytopathology 100:612-618.

We analyzed the avirulence gene AVR-Pital in Japanese rice blast isolates to determine how they gain virulence toward rice cultivars containing the Pita resistance gene. An avirulent isolate, OS99-G-7a (G7a), from a Japanese commercial field contained two paralogs of $A V R$ Pital, designated as AVR-Pital ${ }^{J A}$ and AVR-Pital ${ }^{J B}$. Analysis of virulent, independent mutants derived from G7a, a single avirulent progenitor strain, indicated that AVR-Pital $I^{J A}$ was functional but AVR-Pital ${ }^{J B}$ was

that most of the AVR-Pital genes carried by Japanese isolates were identical to AVR-Pital ${ }^{J A}$ or AVR-Pital ${ }^{J B}$. The relationship between these major paralogs in Japanese isolates and the virulence of the strains carrying them indicate that AVR-Pital ${ }^{J A}$ is functional but $A V R$-Pital ${ }^{J B}$ is not, as is the case in G7a. Isolates that show virulence toward rice cultivars containing the Pita gene are presumed to have evolved virulence from avirulent origins via loss of AVR-Pital ${ }^{J A}$, except for one case in which virulence resulted from a base substitution. In this study, we discuss the properties and specificities of Japanese rice blasts that relate to virulence against Pita-containing rice. Furthermore, we present a method to amplify AVR-Pital ${ }^{J A}$ and AVR-Pital ${ }^{J B}$ separately and, specifically, to monitor functional AVR-Pital in Japan.
\end{abstract} nonfunctional. The most frequent mutation was loss of AVR-Pital ${ }^{J A}$. Analyses of field isolates collected from diverse areas in Japan revealed

Rice blast disease is caused by Magnaporthe oryzae (anamorph Pyricularia oryzae) and is the most economically important rice disease worldwide. More than 40 resistance genes to rice blast disease have been identified and some have been bred into new cultivars (3). However, a number of resistant rice cultivars have become susceptible to the disease within 1 to 6 years of commercial production (2). These disease outbreaks were caused by spontaneous virulent blast mutants that arose as a result of the selection pressure imposed by resistant rice cultivars (17).

In Japan, almost all rice blast isolates from paddy fields are avirulent against rice cultivars containing the Pita gene ( $>99 \%$ of all blast isolates) (15). Thus, Pita is one of the most useful resistance genes in Japan, and several Pita-containing isogenic lines have been bred $(1,23)$. However, production of new cultivars containing the Pita gene increases the possibility that new virulent mutants will arise, and the new cultivars will become susceptible.

Corresponding author: M. Takahashi; E-mail address: mamitaka@affrc.go.jp

* The $\boldsymbol{e}$-Xtra logo stands for "electronic extra" and indicates that the online version contains a figure showing sequence comparisons among AVR-Pital in O-137, AVRPital $^{J A}$, and AVR-Pital ${ }^{J B}$.

doi:10.1094/PHYTO-100-6-0612

(C) 2010 The American Phytopathological Society
Additional keywords: Magnaporthe oryzae, Pyricularia oryzae.
Pita and its corresponding avirulence gene, AVR-Pita, have been identified and characterized. Pita is located on chromosome 12 of the rice genome and encodes a protein with a nucleotidebinding site and a leucine-rich repeat (LRR) domain (4). AVRPita is located in the telomeric region of chromosome 3 and encodes a putative zinc metalloprotease (20). The translated protein, Pita, binds directly to AVR-Pita and the interaction between them induces the defense response (10). Recently, it was found that AVR-Pita constitutes a gene family with functional and nonfunctional avirulence genes with similar sequences (AVR-Pita2 and avr-Pita3) in M. oryzae isolated from diverse hosts. AVR-Pita was renamed AVR-Pital (12).

The AVR-Pital locus of five blast isolates from diverse hosts has been described in detail elsewhere (12) but can be summarized as follows. In the rice blast isolate O-137, the solo longterminal repeat (LTR) of a retrotransposon, RETRO7-1 (5), is located in the $5^{\prime}$ flanking region of the AVR-Pital promoter, and a telomeric repeat is located $48 \mathrm{bp}$ downstream from the stop codon of AVR-Pital in its $3^{\prime}$ flanking region. In the rice blast isolate 7015 (5), the LTR of RETRO6-1 (5) is joined to a partial solo LTR of RETRO7-1 and is located in the 5' flanking region of the AVRPital promoter. In its $3^{\prime}$ flanking region, there are three linked solo LTRs, one from each of three retrotransposons: RETRO7-1, Pyret (19), and RETRO6-1. In the Digitaria isolate G-1, the 5' 
truncated RETRO6-1 adjoins a telomeric repeat and a partial solo LTR of RETRO7-1 in the 5' flanking region of the AVR-Pital promoter, and the $3^{\prime}$ flanking region contains RETRO7-1. The telomeric repeat is located $1,689 \mathrm{bp}$ upstream from the start codon of AVR-Pital. In isolate G-223 from Pennisetum spp., structures around AVR-Pital are similar to those of G-1. However, the telomeric region and internal region of RETRO6-1 in G-1 are replaced by a sequence that was predicted to be nonfunctional. It was presumed that a telomeric repeat lies within a $30-\mathrm{kbp}$ distance of the G223 AVR-Pital. In Pennisetum spp. isolate G-78, the partial solo LTR of RETRO6-1 and the Pot2-like transposable element (612 bp upstream of solo LTR) are located in the $5^{\prime}$ flanking region of the AVR-Pital promoter, while the $3^{\prime}$ flanking region contains RETRO7-1 with duplicated termini of the LTR. Thus, all studied AVR-Pital genes are associated with sequences for transposable elements, which are dispersed throughout the genomes, and three of the five AVR-Pital genes are located near telomeric repeats. In addition, the flanking regions of the five studied AVR-Pital showed differences in the positions of telomeric repeats and in gene organization (i.e., the types, numbers, and lengths of LTRs and transposable elements).

Previously, rice blast mutants that gained virulence against Pita-containing rice cultivars have been isolated under laboratory conditions and their genetic alterations examined. There are various changes in the AVR-Pital gene, including point mutations, transposon insertions, and deletions ranging in size from $100 \mathrm{bp}$ to $12.5 \mathrm{kbp}$, which enable strains to gain virulence against Pitacontaining rice $(11,20,24)$. Likewise, analysis of field isolates from the United States showed that transposon insertion and deletions of AVR-Pital resulted in a gain of virulence against Pitacontaining cultivars. In the United States, isolates that were avirulent against Pita-containing rice always contained AVR-Pital, which was confirmed by polymerase chain reaction (PCR) amplification (29).

In this study, we aimed to clarify how Japanese rice blast gains virulence against Pita-containing rice, and to develop a specific PCR method that can be used to monitor functional AVR-Pital. Rice blast strains that are virulent against Pita-containing rice are very rarely isolated from the restricted area in which Pitacontaining rice is cultivated in Japan. We investigated the presence and types of AVR-Pital in Japanese field isolates, including virulent isolates. Our results differ from those of field isolates in the United States. Here, we report the specificity related to Pitamediated resistance in Japanese rice blast strains, and we describe a PCR method to detect AVR-Pital based on its specificity.

\section{MATERIALS AND METHODS}

Rice blast isolates. We used OS99-G7-a (G7a) and 13 independent virulent mutants derived from it to clarify the DNA alterations that affect virulence in Pita-containing cultivars. G7a is an isolate obtained from a commercial rice field in Japan. It has a unique DNA sequence that is used to identify it (8). All the mutants were independent and developed spontaneously. Of the 13 mutants, 5 (S06-1-1, S06-11-1, S06-13-1, S06-14-1, and S071) were isolated from the Pita-containing cultivar, Sasanishiki BL6 $(1,22)$, inoculated with conidia of G7a. Fifty rice plants at approximately the seven- to eight-leaf stage were inoculated with $30 \mathrm{ml}$ of conidial suspension $\left(8.0 \times 10^{4}\right.$ conidia/ml $)$ and incubated in a moist chamber at $26^{\circ} \mathrm{C}$ for $20 \mathrm{~h}$. After 10 days, susceptible lesions were collected (26). We isolated 8 of the 13 mutants from experimental paddy fields with severe infestations of $\mathrm{G} 7$ a rice blast. The mutants P06-1, P07-2-1, P07-3-1, and P07-5-1 were isolated from Sasanishiki BL6 in paddy fields that were planted in alternate rows with the susceptible rice cultivar (Koshihikari or Sasanishiki) and Sasanishiki BL6 (22). The mutants 01-1-1-1, 0126-2-1, 01-27-1-1, and 01-95-1-1 were isolated from a nursery in Yashiromochi (21), from a seedling case that was placed in a paddy field infested with G7a. This paddy field was planted with Niigata Koshihikari BL1 (Pia), Koshihikari BL2 (Pii), Koshihikari BL3 (Pita-2), and Koshihikari BL4 (Piz) (9). To cause epidemics, rice plants infected with G7a were transferred to paddy fields. Susceptible lesions were collected only once, when they were first found. This avoided harvest of lesions formed by replicated mutants. G7a-derived mutants were identified by PCR (8).

In addition to $\mathrm{G} 7 \mathrm{a}$, we used 32 Japanese field isolates collected in 19 prefectures in this study. Of the 32 isolates, 8 were virulent and 24 were avirulent. Of these 32 isolates, 14 isolates, including all the virulent isolates, were analyzed by sequencing and Southern hybridization. The 18 isolates that were all avirulent were analyzed by PCR amplification to detect the presence of $A V R$-Pital ${ }^{J A}$ or AVR-Pital ${ }^{J B}$. IAI-1, P07-10, SYaP1, and SYaF1 were isolated from an experimental field planted with the rice cv. Yashiromochi or Sasanishiki BL6. We isolated 031713 and 031719 from the rice cv. Koshihikari in a commercial field in Niigata prefecture. Sixteen rice blast strains (0115006 to 0132034) were isolated from commercial fields to investigate races in Japan in 2001 (15). GF0S8-1-1, 0528-2, Ken 54-20, Hoku 1, TH68-140, Ken 54-04, Kyu 92-22, 24-22-1-1, Kyu9439013, and Kyu89-246 were stored for genetic analyses at the National Agricultural Research Center (NARC) $(7,14)$. Information on the 32 isolates besides G7a, their race codes in Japan $(13,25)$, and their pathogenic or nonpathogenic interactions with rice cv. Yashiromochi are shown in Table 1.

A single conidium was isolated from lesions that we had collected, and was cultured on oatmeal medium. Conidia formation was induced by brushing of mycelia, and then incubating the culture for 3 days under white fluorescent lights. To retest the virulence of rice blast strains against Pita-containing rice, conidia

TABLE 1. Japanese rice blast isolates used in this study

\begin{tabular}{|c|c|c|c|c|}
\hline \multirow[b]{2}{*}{ Isolate } & \multirow[b]{2}{*}{ Reactions $^{\mathrm{a}}$} & \multicolumn{2}{|c|}{ Origin } & \multirow[b]{2}{*}{ Race $^{b}$} \\
\hline & & Prefecture & Year & \\
\hline IAI-1 & + & Ibaraki & 2006 & 117.1 \\
\hline P07-10 & + & Niigata & 2007 & 307.0 \\
\hline SYaP1 & + & Shimane & 2007 & 137.1 \\
\hline SYaF1 & + & Shimane & 2007 & 307.0 \\
\hline 031713 & + & Niigata & 2007 & 103.0 \\
\hline 0132034 & + & Shimane & 2001 & 101.0 \\
\hline GFOS8-1-1 & + & Gifu & 1993 & 303.0 \\
\hline $0528-2$ & + & Akita & 1976 & 333.1 \\
\hline OS99-G-7a & - & Saga & 1999 & 007.0 \\
\hline 0115006 & - & Niigata & 2001 & 001.0 \\
\hline 0115018 & - & Niigata & 2001 & 003.0 \\
\hline 0116020 & - & Toyama & 2001 & 047.0 \\
\hline 0116030 & - & Toyama & 2001 & 001.0 \\
\hline 0117011 & - & Ishikawa & 2001 & 001.0 \\
\hline 0117012 & - & Ishikawa & 2001 & 041.0 \\
\hline 0118009 & - & Fukui & 2001 & 037.1 \\
\hline 0118025 & - & Fukui & 2001 & 003.0 \\
\hline 0125029 & - & Shiga & 2001 & 003.0 \\
\hline 0125030 & - & Shiga & 2001 & 007.0 \\
\hline 0128112 & - & Hyogo & 2001 & 007.0 \\
\hline 0128123 & - & Hyogo & 2001 & 001.0 \\
\hline 0130114 & - & Wakayama & 2001 & 007.0 \\
\hline 0131041 & - & Tottori & 2001 & 001.0 \\
\hline 0132032 & - & Shimane & 2001 & 001.0 \\
\hline 031719 & - & Niigata & 2007 & 003.0 \\
\hline Hoku 1 & - & Hokkaido & 1947 & 007.0 \\
\hline TH68-140 & - & Yamagata & 1968 & 035.1 \\
\hline Ken 54-04 & - & Gifu & 1954 & 003.0 \\
\hline $24-22-1-1$ & - & Mie & 1994 & 037.1 \\
\hline Ken54-20 & - & Yamaguchi & 1954 & 003.0 \\
\hline Kyu9439013 & - & Kochi & 1994 & 047.1 \\
\hline Kyu89-246 & - & Miyazaki & 1989 & 003.0 \\
\hline Kyu92-22 & - & Kumamoto & 1992 & 017.1 \\
\hline
\end{tabular}

a Reactions to Yashiromochi (Pita) $+=$ virulence and $-=$ avirulence.

b According to the method described by Yamada et al. (25) and Kiyosawa (13). 
were inoculated onto Yashiromochi rice plants at approximately the four- to five-leaf stage using a spray method (26).

PCR, cloning, and sequencing. Rice blast isolates were cultured in potato dextrose broth (PDB) for 2 or 3 days and DNA was extracted using an ISOPLANT II kit (Nippon Gene Co. Ltd., Tokyo). PCR was performed using a Gene Amp PCR System 9700 (Applied Biosystems Inc., Foster, CA).

For direct sequence analysis, AVR-Pital was amplified with the primers AVR-PitaN89 (5'-CCGCCTTTATTGGTTTAATTCGGA TA-3') and BamHIAVR-PitaR1 (5'-ATGGATCCTCCATTCC AACACTAACG-3'). For PCR, the 50- $\mu$ l reaction mixture contained $\approx 50 \mathrm{ng}$ of genomic DNA, 25 pmol each primer, $0.2 \mathrm{mM}$ dNTP, $5 \mu \mathrm{l}$ of $10 \times$ buffer, and 1.25 units of ExTaq polymerase (Takara Bio. Inc., Otsu, Shiga, Japan). The PCR cycling program consisted of initial denaturation for $5 \mathrm{~min}$ at $94^{\circ} \mathrm{C} ; 30$ cycles of 1 min of denaturation at $94^{\circ} \mathrm{C}, 1 \mathrm{~min}$ of annealing at $55^{\circ} \mathrm{C}$ and $90 \mathrm{~s}$ of extension at $72^{\circ} \mathrm{C}$; followed by final extension for $7 \mathrm{~min}$ at $72^{\circ} \mathrm{C}$. Amplified fragments were purified using a QIA quick PCR purification kit (Qiagen Inc., Valencia, CA).

For cloning, AVR-Pital of G7a was amplified with the primers BamHIAVR-PitaL1 (5'-AAGGATCCGGAGCCGAGTCGTTCT GA-3') and BamHIAVR-PitaR1. For PCR, the 50- $\mu$ l reaction mixture contained $\approx 50 \mathrm{ng}$ of genomic DNA, 25 pmol each primer, $0.2 \mathrm{mM}$ dNTP, $1 \mathrm{mM} \mathrm{MgSO} 4,5 \mu \mathrm{l}$ of $10 \times$ buffer, and 1 unit of KOD Plus polymerase (Toyobo Co. Ltd., Osaka, Japan). The PCR cycling program consisted of initial denaturation for $2 \mathrm{~min}$ at $94^{\circ} \mathrm{C} ; 35$ cycles of $15 \mathrm{~s}$ of denaturation at $94^{\circ} \mathrm{C}, 30 \mathrm{~s}$ of annealing at $55^{\circ} \mathrm{C}$, and $90 \mathrm{~s}$ of extension at $68^{\circ} \mathrm{C}$; followed by final extension for $5 \mathrm{~min}$ at $68^{\circ} \mathrm{C}$. Except in G7a, AVR-Pital was amplified using the primers BamHIAVR-PitaN89 (5'-AAGGATCCGACCC GTTTCCGCCTTTATT-3') and HindIIIAVR-PitaR1 (5'-ATAA GCTTTCCATTCCAACACTAACG-3'). PCR was carried out using KOD plus polymerase or Pyrobest polymerase (Takara Bio. Inc.). When KOD plus polymerase was used, the PCR procedure was exactly the same as that used to amplify AVR-Pital from G7a. In the case of Pyrobest polymerase, the 50- $\mu$ PCR reaction mixture contained $\approx 50 \mathrm{ng}$ of genomic DNA, 25 pmol each primer, $0.2 \mathrm{mM}$ dNTP, 2.5 units of Pyrobest polymerase, and $5 \mu \mathrm{l}$ of $10 \times$ buffer. The PCR cycling program consisted of 30 cycles of $10 \mathrm{~s}$ of denaturation at $98^{\circ} \mathrm{C}, 30 \mathrm{~s}$ of annealing at $55^{\circ} \mathrm{C}$, and $60 \mathrm{~s}$ of extension at $72^{\circ} \mathrm{C}$. Amplified fragments were purified using a QIAquick PCR purification kit (Qiagen Inc.) and digested with restriction enzymes corresponding to additional restriction sites in the PCR primers. The fragments were purified from agarose gel slices and cloned into pUC19.

To examine the promoter region of AVR-Pital in S06-14-1, we conducted PCR amplification using three sets of primers, as follows: BamHIAVR-PitaL1 and BamHIAVR-PitaR1; AVR-Pita410-394 (5'-TTATGTAGTTATTAATAATATGGGCC-3') and BamHIAVR-PitaR1; and AVRPita-210-181 (5'-AGTCAAGCTG AAATAATAAATAAGTTGGCG-3') and BamHIAVR-PitaR1. To identify AVR-Pital ${ }^{J A}$ in Ken54-20, we used the primer set AVRPitaN89 and AVR-PitaApaLI (5'-CGTGCACATTGTGCAAA AATAGCATAAC-3'). The PCR procedures for examination of promoter regions in S06-14-1 and for identification of $A V R$ Pital $^{J A}$ in Ken54-20 were the same as those used for amplification of AVR-Pital for direct sequencing.

Sequencing reactions were carried out using the GenomeLab DTCS Quick Start Kit, and were analyzed using a CEQ8000 system according to the manufacturer's instructions (Beckman Coulter Inc., Fullerton, CA). Sequencing primers were as follows: AVR-PitaN89, BamHIAVR-PitaR1, BamHIAVR-PitaL1, AVRPita1161F (5'-TTCACACCGTTGCGATTTCG-3'); AVR-Pita454478 (5'-GTTTATACGTGCGTCCGAGAAGAAG-3'); AVRPita758-733 (5'-CTTACTTGTGAATCCCATCCCATTCG-3'); AVR-Pita1697R (5'-GGTTACCGGCAGTAATTTCC-3'); AVRPita-318-347R (5'-ATTATGCAGGTAATTAGGTCCACTT-3'); and the $B c a \mathrm{BEST}$ sequencing primer RV-M (Takara Bio. Inc.).
The following primers were designed to specifically amplify AVR-Pital ${ }^{\mathrm{JA}}$ : 5'AVR-Pita1 ${ }^{\mathrm{JA}} \mathrm{F}$ (5'-GCTATCACGCCGTTAAAAG TG-3') and 5'AVR-Pita ${ }^{\mathrm{JA}} \mathrm{R}$ (5'-TAGCATAACTGTCGGGG TTTT-3'). To specifically amplify AVR-Pital ${ }^{J B}$, we used $5^{\prime}$ AVR-

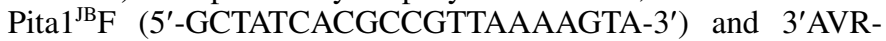
Pita ${ }^{\mathrm{JB}} \mathrm{R}$ (5'-GCATAACTGTCGGGGTTTC-3'). The $3^{\prime}$ terminal nucleotides of these primers corresponded to the nucleotides that differ between AVR-Pital ${ }^{J A}$ and AVR-Pital ${ }^{J B}$. The PCR mixture $(20 \mu \mathrm{l})$ contained $50 \mathrm{ng}$ of genomic DNA, 1 pmol each primer, $2 \mu \mathrm{l}$ of $10 \times$ reaction buffer, $0.2 \mathrm{mM} \mathrm{dNTP}$, and 1.25 units of ExTaq polymerase (Takara Bio. Inc.). The PCR cycling program consisted of an initial denaturation for $7 \mathrm{~min}$ at $94^{\circ} \mathrm{C} ; 30$ cycles of $1 \mathrm{~min}$ of denaturation at $94^{\circ} \mathrm{C}, 1 \mathrm{~min}$ of annealing at $64^{\circ} \mathrm{C}$, and $90 \mathrm{~s}$ of extension at $72^{\circ} \mathrm{C}$; followed by a final extension for $7 \mathrm{~min}$ at $72^{\circ} \mathrm{C}$.

Southern blot hybridization. Rice blast isolates were cultured in PDB for 14 days and genomic DNA was extracted using a DNeasy Plant Maxi kit (Qiagen Inc.). Approximately $3 \mu \mathrm{g}$ of DNA was digested with $\approx 4$ to 12 units of restriction enzymes at $37^{\circ} \mathrm{C}$ overnight. DNA was separated by electrophoresis on a $0.67 \%$ agarose gel and blotted onto a Hybond $\mathrm{N}^{+}$membrane (GE Healthcare, UK Ltd., Buckinghamshire, England) using a BacuGene XL Vacuum Blotting system (GE Healthcare, UK Ltd.). Hybridization was performed using an AlkPhos Direct Labeling and Detection System (GE Healthcare, UK Ltd.) according to the manufacturer's instructions. The fragment amplified from genomic DNA of G7a with BamHIAVR-PitaN89 and BamHIAVR-PitaR1 was digested with BamHI and ApaLI and separated by electrophoresis on a $1 \%$ agarose gel to use as a DNA probe. The probe was hybridized to the blot at $55^{\circ} \mathrm{C}$ overnight.

\section{RESULTS}

AVR-Pital in G7a. AVR-pital genes in G7a were amplified, cloned, and sequenced. Two paralogs were found in G7a. These paralogs shared $>98 \%$ nucleotide sequence identity with $A V R$ Pital cloned from O-137 (20). A phylogenic tree was constructed by the neighbor-joining method using the nucleotide sequences of these two paralogs in G7a and those of other members of the AVR-Pita gene family described by Khang et al. (12). The tree revealed that the two paralogs in G7a were found in the $A V R$ Pital clade, which was separate from the AVR-Pita2 and avrPita3 clades (data not shown). Therefore, the two paralogs in G7a were designated as AVR-Pital ${ }^{J A}$ (accession no. AB520831) and $A V R$-Pital ${ }^{J B}$ (accession no. AB520830). Sequences of AVR-Pital ${ }^{J A}$ and $A V R$-Pital ${ }^{J B}$ were identical except for three nucleotides at positions 303,719 , and 829 bp (numbering is relative to the translation start site of AVR-Pital ${ }^{J A}$ or AVR-Pital ${ }^{J B}$ ). The three nucleotides that differed were $\mathrm{G}, \mathrm{A}$, and $\mathrm{A}$ in AVR-Pital ${ }^{\mathrm{JA}}$ and $\mathrm{A}$, $\mathrm{G}$, and $\mathrm{G}$ in $A V R-$ Pital $^{J B}$. These differences in DNA sequences were predicted to affect the amino acid sequences as follows: D (83), Y (192), and K (207) in AVR-Pita1 ${ }^{\mathrm{JA}}$; and N (83), C (192), and $\mathrm{R}$ (207) in AVR-Pita1 ${ }^{\mathrm{JB}}$. The amino acids D (83), Y (192), and $\mathrm{K}$ (207) in AVR-Pital ${ }^{\mathrm{JA}}$ were identical to the corresponding residues in AVR-Pita1 in O-137. The sequences of promoter regions of the two AVR-Pital genes in G7a were also determined. The upstream sequences (approximately -492 to $-1 \mathrm{bp}$ ) of $A V R$ Pital $^{J A}$ and $A V R$-Pital ${ }^{J B}$ were identical to each other and were $>98 \%$ identical to those of AVR-Pital in O-137.

Southern blot analyses were carried out to identify the copy number of AVR-Pital in G7a (Fig. 1). We used ApaLI to digest genomic DNA and to produce the probe for the blot, because its cleavage site is located in the $3^{\prime}$ terminal regions of AVR-Pital ${ }^{J A}$ or $A V R-$ Pital $^{J B}$. This avoids detecting a band containing more than two AVR-Pital sequences. Two bands were detected in all lanes, regardless of whether the DNA was digested with KpnI, $A p a \mathrm{LI}$, or both enzymes. The two bands migrated faster in the lane of the double digestion compared with those in the lanes of 
single digestions. This result indicates that both ends of each band in the lane of the double digestion had been digested by KpnI and $A p a \mathrm{LI}$, and that each band contained one AVR-Pital paralog. This result supported the idea that G7a contains two paralogs. We tried to amplify the AVR-Pita2 in G7a as described by Khang et al. (12); however, we did not obtain an AVR-Pita2 fragment.

AVR-Pital in G7a-derived mutants. To clarify whether two paralogs were carried over into 13 G7a-derived mutants, we amplified and directly sequenced AVR-Pital paralogs, taking particular note of the different nucleotides at 303, 719, and 829 . In the mutant strains 01-95-1-1, S06-13-1, and S06-14-1, two signals of $\mathrm{G}$ and $\mathrm{A}$ were detected at positions corresponding to the three different nucleotides deduced from sequence data. In the other mutants, only one signal was detected at the position corresponding to the three different nucleotides. In addition, their amplicons shared $100 \%$ sequence identity with AVR-Pital ${ }^{J B}$. These results suggest that 01-95-1-1, S06-13-1, and S06-14-1 retain both $A V R$-Pital ${ }^{J A}$ and AVR-Pital ${ }^{J B}$, but the other 10 mutants retain only AVR-Pital ${ }^{J B}$ (Table 2).

Next, we cloned and sequenced amplicons of 01-95-1-1, S0613-1, and S06-14-1 (Table 2). In all three mutants, AVR-Pital ${ }^{J B}$ was unchanged. AVR-Pital ${ }^{J A}$ was unchanged only in the S06-14-1 mutant. In 01-95-1-1, AVR-Pital ${ }^{J A}$ had a tandem duplication of 31 bp from 795 to 825 bp. In S06-13-1, AVR-Pital ${ }^{J A}$ lacked a T at the 30-bp position. Both changes were predicted to cause a frame shift resulting in C-terminal-truncated AVR-Pita $1^{\mathrm{JA}}$. Because $A V R$-Pital ${ }^{J A}$ and AVR-Pital ${ }^{J B}$ were unchanged in S06-14-1, their promoter regions were also analyzed. Fragments were amplified from genomic DNA of S06-14-1 using three primer sets: BamHIAVR-PitaL1 and BamHIAVR-PitaR1, AVR-Pita-410-394 and BamHIAVR-PitaR1, and AVRPita-210-181 and BamHIAVRPitaR1. The resulting fragments were sequenced directly. Using the primer set AVRPita-210-181/BamHIAVR-PitaR1, both AVR-

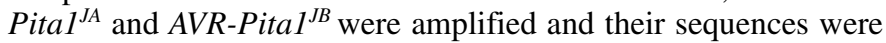
unchanged from those in G7a. However, when the primer sets
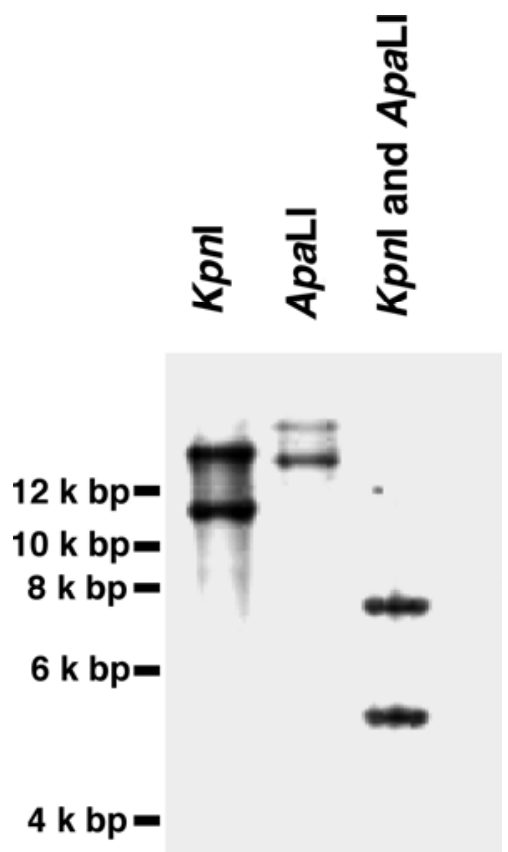

Fig. 1. Southern blot analysis of AVR-Pital in rice blast isolate OS99-G-7a. Genomic DNA was digested with KpnI, ApaLI, or both. Products were separated by electrophoresis on a $0.67 \%$ agarose gel and transferred to a nylon membrane. A labeled fragment that was amplified with BamHIAVR-PitaN89 and BamHIAVR-PitaR1 and digested with BamHI and ApaLI was used as a probe. Hybridization was carried out using an AlkPhos Direct Labeling and Detection System according to the manufacturer's instructions (GE Healthcare, UK Ltd.). Sizes of markers are shown at left.
BamHIAVR-PitaL1/BamHIAVR-PitaR1 and AVR-Pita-410-394/ BamHIAVR-PitaR1 were used, AVR-Pital ${ }^{J B}$ was amplified but $A V R$-Pital ${ }^{J A}$ was not. Therefore, the primers BamHIAVR-PitaL1 and AVR-Pita-410-394 could not amplify AVR-Pital ${ }^{J A}$. The sequence analysis of PCR fragments suggested that the region containing the sequences of these primers is deleted from the promoter of AVR-Pital ${ }^{J A}$ in S06-14-1.

We carried out Southern blot analyses to confirm the copy number of AVR-Pital (Fig. 2). Two bands were detected in the lanes of 01-95-1-1, S06-13-1, and G7a. A band approximately 5 $\mathrm{kbp}$ in length, which corresponds to the lower band in G7a, was absent from the lanes of the other 11 mutants. These results supported the idea that two paralogs were carried over into 01-951-1 and S06-13-1, and that AVR-Pital ${ }^{J A}$ was deleted from the genomes of 10 mutants. However, a hybridization analysis of

TABLE 2. Variations in AVR-Pital in OS99-G-7a-derived mutants ${ }^{\mathrm{a}}$

\begin{tabular}{lll}
\hline & \multicolumn{2}{c}{ Mutation of } \\
\cline { 2 - 3 } Isolate & \multicolumn{1}{c}{$A V R$-Pital } & \\
\hline 01-1-1-1 & Deletion of gene & NVR-Pital ${ }^{J B}$ \\
$01-26-2-1$ & Deletion of gene & No change \\
$01-27-1-1$ & Deletion of gene & No change \\
$01-95-1-1$ & 31 bp of duplication & No change \\
S06-1-1 & Deletion of gene & No change \\
S06-11-1 & Deletion of gene & No change \\
S06-13-1 & Deletion of thymine base & No change \\
S06-14-1 & Partial deletion of promoter & No change \\
P06-1 & Deletion of gene & No change \\
S07-1 & Deletion of gene & No change \\
P07-2-1 & Deletion of gene & No change \\
P07-3-1 & Deletion of gene & No change \\
P07-5-1 & Deletion of gene & No change \\
\hline
\end{tabular}

${ }^{a}$ Confirmed by sequencing of amplified fragment with AVR-PitaN89 and BamHIAVR-PitaR1 or BamHIAVR-PitaN89 and HindIIIAVR-PitaR1, and Southern blot hybridization (except for mutation of S06-14-1). Deletion of AVR-Pital ${ }^{J A}$ promoter in S06-14-1 was confirmed by amplification with specific primers for the promoter region and Southern blot hybridization.

b Tandem duplication from 795 to $895 \mathrm{bp}$ (numbering is relative to the translation start site of AVR-Pital ${ }^{J A}$ or AVR-Pital ${ }^{J B}$ ).

${ }^{\mathrm{c}}$ Located at 30-bp position.
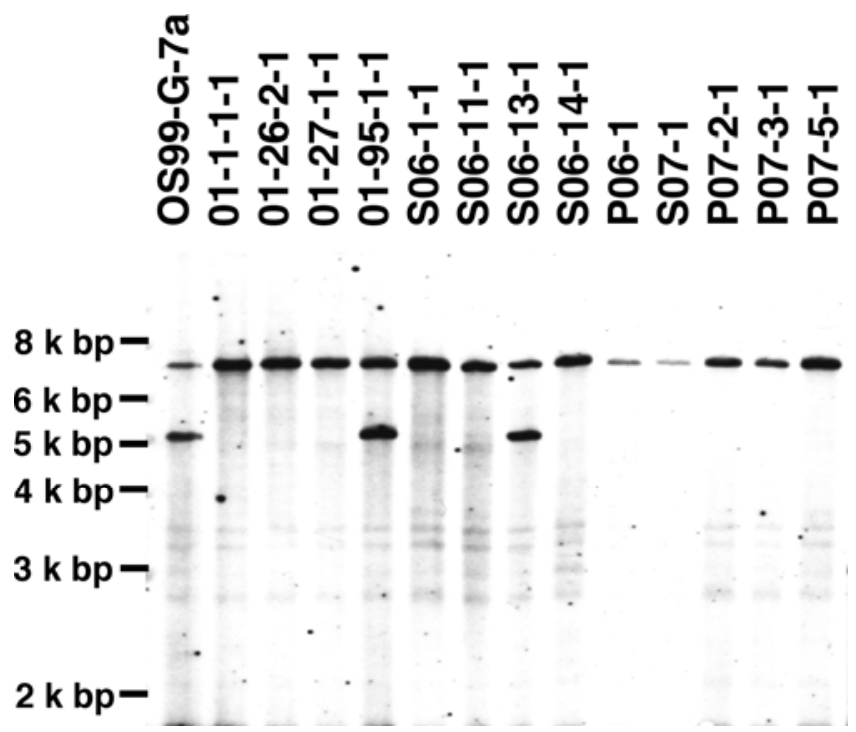

Fig. 2. Southern blot analysis of AVR-Pital in virulent mutants derived from OS99-G-7a. Genomic DNA was digested with KpnI and ApaLI, separated by electrophoresis on a $0.67 \%$ agarose gel, and then transferred to a nylon membrane. A labeled fragment that was amplified with BamHIAVR-PitaN89 and BamHIAVR-PitaR1 and digested with BamHI and ApaLI was used as a probe. Hybridization was performed with the AlkPhos Direct Labeling and Detection System (GE Healthcare, UK Ltd.) according to the manufacturer's instructions. Names of mutants are shown above photographs. Sizes of markers are shown at left. 
S06-14-1 suggested the presence of one paralog, which did not agree with the sequence analysis result. To consider the possibility that there were two overlapping bands, we digested genomic DNA of S06-14-1 with ApaLI and ApaI, because the $A p a \mathrm{I}$ site lies in the deleted region of the promoter in $A V R$ Pital $^{J A}$. As expected, the Southern blot analysis revealed two bands in the lane of S06-14-1 (Fig 3). The 1.25-kbp band was identical in size to the band in G7a and was predicted to contain $A V R$-Pital ${ }^{J B}$. The other band was predicted to contain $A V R$ Pita $^{J A}$ with a partial deletion in its promoter.

Taken together, these results demonstrated that AVR-Pital ${ }^{J B}$ was unchanged in G7a-derived mutants but $A V R$-Pital $I^{J A}$ or its promoter was altered.

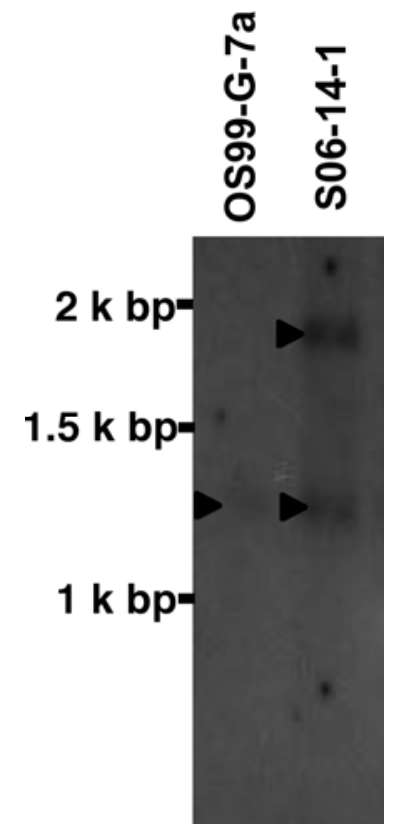

Fig. 3. Southern blot hybridization of S06-14-1. Genomic DNA was digested with ApaI and ApaLI, separated electrophoretically on a $0.67 \%$ agarose gel, and transferred to a nylon membrane. A labeled fragment that was amplified with BamHIAVR-PitaN89 and BamHIAVR-PitaR1 and digested with BamHI and $A p a$ LI was used as a probe. Hybridization was performed using the AlkPhos Direct Labeling and Detection System (GE Healthcare, UK Ltd.) according to the manufacturer's instructions. Sizes of markers are shown at left.

TABLE 3. AVR-Pita1 paralogs in 14 Japanese isolates ${ }^{\mathrm{a}}$

\begin{tabular}{lcccc}
\hline Isolate & AVR-Pital $^{J A b}$ & AVR-Pital $^{J B c}$ & AVR-Pital $^{J C \mathrm{~d}}$ & AVR-Pita $^{J \mathrm{e}}$ \\
\hline IAI-1 & - & + & - & - \\
031713 & - & + & - & - \\
P07-10 & - & + & - & - \\
0132034 & - & + & - & - \\
SYaP1 & - & + & - & - \\
SYaF1 & - & + & + & - \\
GFOS8-1-1 & - & - & - & - \\
$0528-2$ & - & + & - & - \\
031719 & + & + & - & - \\
0132032 & + & + & - & - \\
0131041 & + & + & - & - \\
Ken54-20 & + & - & - & - \\
Hoku 1 & + & - & - & - \\
TH68-140 & - & + & - & + \\
\hline
\end{tabular}

${ }^{\text {a Symbols: }-=\text { not identified and }+=\text { identified by sequencing of amplified }}$ fragments with primer sets AVR-PitaN89 and BamHIAVR-PitaR1 or BamHIAVR-PitaN89 and HindIIIAVR-PitaR1.

${ }^{\mathrm{b}}$ Accession no. AB520831.

c Accession no. AB520830.

d Paralog is identical to AVR-Pital ${ }^{J A}$ in G7a-derived mutant (S06-13-1) (i.e., $A V R$-Pital ${ }^{J A}$ lacks T at $30 \mathrm{bp}$ ).

${ }^{\text {e }}$ Sequence identical to that of AVR-Pital ${ }^{J A}$, except for C at the 411-bp position.
AVR-Pita1 in Japanese field isolates. We amplified and directly sequenced the AVR-Pital gene from 14 isolates collected from diverse areas in Japan. However, the sequences of a virulent isolate and four avirulent isolates suggested that they contained more than two paralogs. Therefore, their amplicons were cloned and sequenced (Table 3 ). In 14 isolates, paralogs were identical to $A V R$-Pital ${ }^{J A}$ or $A V R$-Pital ${ }^{J B}$, except for the paralogs in SYaF1 and TH68-140. Of the eight virulent isolates, one lacked AVR-Pital (GFOS8-1-1), six contained only AVR-Pital ${ }^{J B}$, and one contained $A V R-$ Pital $^{J B}$ and a paralog designated as AVR-Pital ${ }^{J C}$, which was identical to the altered $A V R$-Pital ${ }^{J A}$ in S06-13-1 (SYaF1). Of the six avirulent isolates, two contained only AVR-Pital ${ }^{J A}$, three contained both AVR-Pital ${ }^{J A}$ and AVR-Pital ${ }^{J B}$, and one isolate, TH68140, contained AVR-Pital $I^{J B}$ and a paralog designed as AVR-Pital ${ }^{J D}$, which had a sequence identical to that of $A V R$-Pital ${ }^{J A}$ except for one base at the 411-bp position (Table 3 ). The cytidine base at the 411-bp position in AVR-Pital ${ }^{J D}$ changes the amino acid sequence, resulting in Q (119).

Southern blot analyses were carried out to confirm the copy number of AVR-Pital in each isolate (Fig. 4). No bands were detected in GFOS8-1-1, one band was detected in six virulent isolates and an avirulent isolate, and two bands were detected in a virulent isolate and five avirulent isolates. The copy numbers determined from Southern blot analyses agreed with the results of sequence analyses, except in Ken 54-20. Two bands were detected in the lane of Ken 54-20, although only AVR-Pital ${ }^{J A}$ was amplified. DNA of Ken 54-20 was separated by agarose electrophoresis and the bands were extracted from agarose gel slices; then, AVRPital genes were amplified and sequenced. The results showed that both bands corresponded to AVR-Pital ${ }^{J A}$ in Ken 54-20.

Because almost all Japanese isolates contained AVR-Pital ${ }^{J A}$ and $A V R$-Pital $1^{J B}$, a method for selective amplification of AVR-Pital ${ }^{J A}$ and AVR-Pital ${ }^{J B}$ was developed. Ken 54-20 and P06-1, which contained only AVR-Pital ${ }^{J A}$ and AVR-Pital ${ }^{J B}$, respectively, were used to develop optimal conditions for specific amplification. We tested six primer sets that were designed using nucleotides that differed between $A V R$-Pital ${ }^{J A}$ and $A V R$-Pital ${ }^{J B}$. Two primer sets, $5^{\prime}$ AVR-Pita $1^{\mathrm{JA}} \mathrm{F} / 3^{\prime}$ AVR-Pita $1^{\mathrm{JA}} \mathrm{R}$ and $5^{\prime}$ AVR-Pita ${ }^{\mathrm{JB}} \mathrm{F} / 3^{\prime}$ AVRPita $1^{\mathrm{JB}} \mathrm{R}$, were the most useful for paralog-specific amplification.

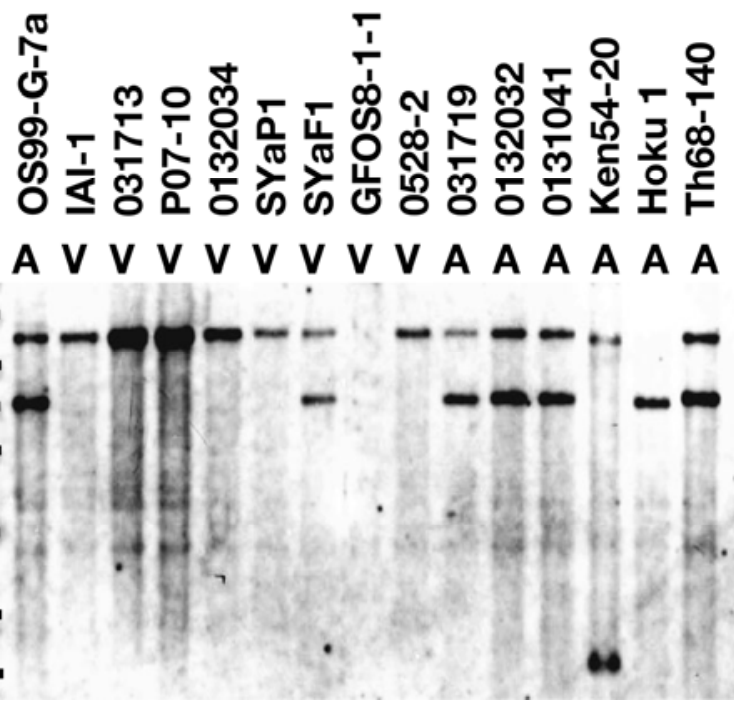

Fig. 4. Southern blot analysis of AVR-Pital in field isolates collected from diverse areas in Japan. Genomic DNA was digested with KpnI and ApaLI, separated electrophoretically on a $0.67 \%$ agarose gel, and then transferred to a nylon membrane. A labeled fragment that was amplified with BamHIAVRPitaN89 and BamHIAVR-PitaR1 and digested with BamHI and ApaLI was used as a probe. Hybridization was performed using the AlkPhos Direct Labeling and Detection System (GE Healthcare, UK Ltd.) according to the manufacturer's instructions. Names of isolates are shown above photographs. Sizes of markers are shown at left. A, avirulent; V, virulent. 
Annealing at $64^{\circ} \mathrm{C}$ enabled each primer set for AVR-Pital ${ }^{J A}$ and $A V R$-Pital ${ }^{J B}$ to amplify AVR-Pital ${ }^{J A}$ in Ken54-20 and AVR-Pital ${ }^{J B}$ in P06-1 exclusively (Fig. 5). We used this method to investigate 18 avirulent isolates, 13 of which were obtained from commercial fields in 2001 (15) and 5 of which were stored at NARC (7). The result revealed that 17 isolates contained both $A V R-P i t a I^{J A}$ and $A V R$-Pital ${ }^{J B}$ and 1 contained only AVR-Pital ${ }^{J A}$ (Fig. 5).

\section{DISCUSSION}

$A V R$-Pital ${ }^{J B}$ was retained in all G7a-derived mutants whereas $A V R$-Pital $I^{J A}$ was deleted or altered. This result indicates that $A V R$ Pital $^{J A}$ is functional in G7a but $A V R$-Pital ${ }^{J B}$ is not. The presence of $A V R-P i t a l^{J B}$ in seven virulent field isolates confirms that $A V R$ $P_{i t a l}{ }^{J B}$ is not functional in those isolates, just as it is not functional in G7a. Furthermore, our results suggest that AVR-Pital ${ }^{J A}$ and AVR-Pital ${ }^{J D}$ would be functional in avirulent isolates. Gene expression confirmed that the upstream region $(-489$ to $-9 \mathrm{bp})$ of $A V R$-Pital functions fully as a promoter (12). Sequence analyses showed $100 \%$ identity of promoter sequences between $A V R$ Pital $^{J A}$ and $A V R$-Pital ${ }^{J B}$, which suggests that the transcriptional activity is the same in these two genes. The fact that AVR-Pital ${ }^{J B}$ is nonfunctional may be due to differences in amino acid residues.

Analyses of G7a-derived mutants showed that the most frequent mutation was the loss of AVR-Pital ${ }^{J A}$. In addition, PCR and sequence analyses confirmed that 38 G7a-derived mutants lacked AVR-Pital ${ }^{J A}$ (data not shown), except for those shown in Table 2. Likewise, Orbach et al. (20) reported that the most frequent mutation by which avirulent isolates became virulent in rice cultivars containing the Pita gene was the loss of AVR-Pital. The high frequency of gene loss of this gene probably resulted from its telomeric location. Except for SaYF1, virulent isolates did not carry AVR-Pital paralogs or carried only AVR-Pital ${ }^{J B}$. On the other hand, avirulent isolates, except for TH68-140, carried only $A V R$-Pital $I^{J A}$ or carried both AVR-Pital ${ }^{J A}$ and AVR-Pital ${ }^{J B}$. These results suggest that loss of $A V R-P i t a l^{J A}$ is the most prominent mechanism of acquiring virulence against rice cultivars containing the Pita gene in Japanese rice blast populations.

AVR-Pital was originally reported as a single-copy gene by analysis of O-137 (20). A number of isolates in the United States and Cameroon showed two bands in hybridization analyses of AVR-Pital (12,29); however, the effects of two genes on pathogenesis in Pita-containing rice remained unclear. This study clarifies impacts of two AVR-Pital genes by analyzing a Japanese isolate carrying two paralogs and its mutants.
In U.S. isolates, virulence or avirulence against a Pita-containing rice cultivar could be determined using PCR amplifications using a primer set that was designed from the promoter sequence of AVR-Pital and sequences from the $3^{\prime}$ side of $A V R$ Pital (29). In Japanese isolates, however, this PCR amplification is ineffective because both avirulent and virulent isolates contain $A V R$-Pital $I^{J B}$, and the primer sets used in that study could amplify both of the AVR-Pital ${ }^{J A}$ and AVR-Pital ${ }^{J B}$ paralogs. In this study, we developed a method to selectively amplify $A V R$-Pital $I^{J A}$ and $A V R$-Pital $I^{J B}$. This method will be useful for predicting virulence of rice blast fungal strains in Japan. The amino acid sequence of AVR-Pita1 in an avirulent isolate from the United States, A347, is identical to that of AVR-Pita $1^{\mathrm{JB}}$. The reason why AVR-Pita1 of A347 was functional in a previous study (29) remains unclear. One possibility is that $\mathrm{A} 347$ has a functional avirulence gene such as AVR-Pita2 as well as the nonfunctional AVR-Pital.

Isolates from the United States show higher diversity of $A V R$ Pital than Japanese isolates (29). Don and coworkers (6) investigated the population structure of Japanese isolates by DNA fingerprinting using the probes MAGGY and MGR586, and showed that genetic diversity was lost in the period between 1960 and 1976. In Japan, there are two lineages, JL1 and JL2, with 97\% of Japanese field isolates in the JL1 lineage. Our analysis suggested that the majority of the avirulent field isolates in Japan carried both AVR-Pital ${ }^{J A}$ and AVR-Pital ${ }^{J B}$. Furthermore, Ken 5404, which is in the JL1 lineage (6), contains AVR-Pital ${ }^{J A}$ and $A V R$-Pital ${ }^{J B}$, like almost all the avirulent field isolates, but Ken 54-20, which is in the JL2 lineage (6), contains only AVR-Pital ${ }^{J A}$. These results suggest that the JL1 lineage may have carried two paralogs, AVR-Pital $1^{J A}$ and AVR-Pital ${ }^{J B}$. Similar analyses of MGR586 revealed that rice blast isolates in the southern United States can be grouped into eight lineages and those in the Philippines can be grouped into six lineages $(16,28)$. In Japan, the rice blast population is derived from only two lineages. This may explain the low diversity of AVR-Pital in Japanese rice blast isolates.

On the basis of the results in this study, we suggest that the main reason for gain of virulence against Pita-containing rice in the field is deletion of the functional AVR-Pital. Recently, the avirulence genes of rice blast, AVR-Pii, AVR-Pia, and AVR-Pik, were isolated and investigated $(18,27)$. These results demonstrated that the presence or absence of these avirulence genes is consistent with the avirulence or virulence of blast isolates, as is the case in AVR-Pital ${ }^{J A}$. The instability of these newly isolated genes is probably because they are located near telomeric regions

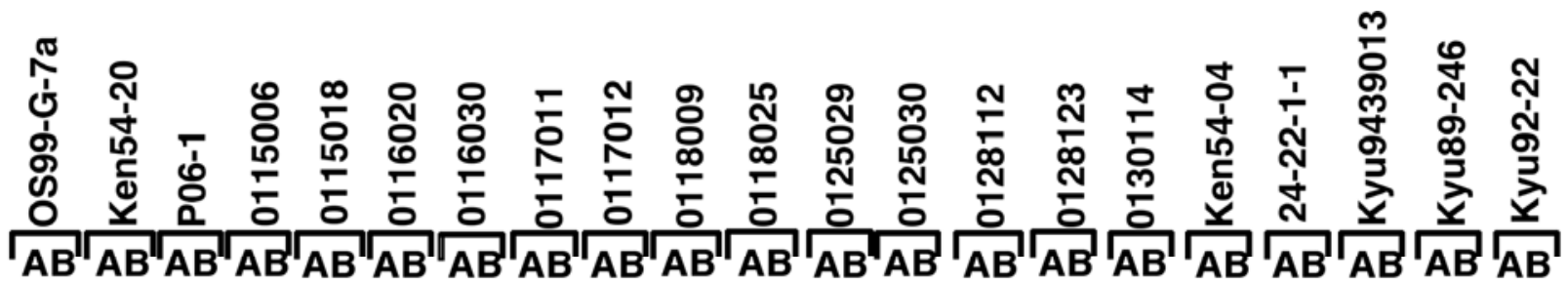

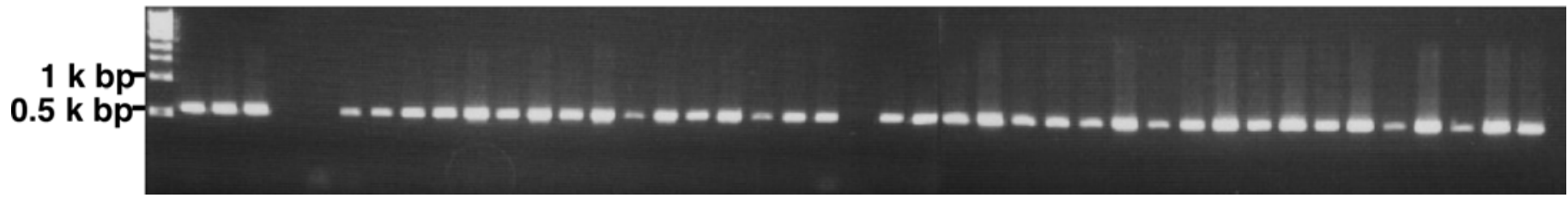

Fig. 5. Specific amplification and identification of $A V R$-Pita $1^{J A}$ and $A V R$-Pital $1^{J B}$ in Japanese isolates. A, Specific primers to amplify AVR-Pita $1^{J A}, 5^{\prime} A V R-P i t a 1^{J A} F$, and $3^{\prime}$ AVR-Pita $1^{\mathrm{JA}}$ R. B, Specific primers to amplify $A V R$-Pita ${ }^{J B}, 5^{\prime}$ AVR-Pita $1^{\mathrm{JB}} \mathrm{F}$, and $3^{\prime}$ AVR-Pita1 ${ }^{\mathrm{JB}} \mathrm{R}$. Names of isolates are shown above photographs. Molecular weight markers are shown at left. For polymerase chain reaction (PCR), the 20- $\mu$ l reaction mixture contained 50 ng of genomic DNA, 1 pmol each primer, $2 \mu \mathrm{l}$ of $10 \times$ reaction buffer, $25 \mathrm{mM}$ dNTP, and 1.25 units of ExTaq polymerase (Takara Bio. Inc.). The PCR cycling program was as follows: initial denaturation for $7 \mathrm{~min}$ at $94^{\circ} \mathrm{C} ; 30$ cycles of $1 \mathrm{~min}$ of denaturation at $94^{\circ} \mathrm{C}, 1 \mathrm{~min}$ of annealing at $64^{\circ} \mathrm{C}$, and $90 \mathrm{~s}$ of extension at $72^{\circ} \mathrm{C}$; and then final extension for $7 \mathrm{~min}$ at $72^{\circ} \mathrm{C}$. Previous sequence analyses confirmed that OS99-G-7a contained both AVR-Pital ${ }^{J A}$ and AVR-Pital ${ }^{J B}$, Ken54-20 contained only AVR-Pital ${ }^{J A}$, and P06-1 contained only AVR-Pita $1^{J B}$. These three isolates were used to validate that the method was paralog specific. All isolates except P06-1 were avirulent against Pita-containing rice. 
or various transposons. The presence of two paralogs suggests that the instability of AVR-Pital could result in either a decrease or an increase in copy number.

We identified a nonfunctional AVR-Pital paralog that is carried in both avirulent and virulent Japanese rice blast isolates. Nonfunctional $A V R$-Pik paralogs were also found in a number of isolates (27). The presence of nonfunctional paralogs also raises the possibility that these avirulence genes have roles other than triggering defense reactions, and are being positively selected for in the field. Furthermore, if these nonfunctional paralogs also have roles other than triggering defense reactions, an isolate carrying one or more nonfunctional paralogs besides a functional paralog may evolve virulence more frequently than one carrying only a functional paralog, which would be completely inactivated by a deletion mutation.

\section{ACKNOWLEDGMENTS}

We thank S. Koizumi for invaluable suggestions and A. Goto for help in sampling of rice blast isolates.

\section{LITERATURE CITED}

1. Abe, S. 2004. Breeding of a blast resistant multiline variety of rice, Sasanishiki BL. JARQ 38:149-154.

2. Asaga, K. 1995. Resistance in rice cultivars. Pages 216-249 in: Rice Blast Disease, 2nd ed. T. Yamanaka and T. Yamaguchi, eds. Yokendo Co. Ltd., Tokyo. (In Japanese)

3. Ashikawa, I., Hayashi, N., Yamane, H., Kanamori, H., Wu, J., Matsumoto, T., Ono, K., and Yano, M. 2008. Two adjacent nucleotide-binding siteleucine-rich repeat class genes are required to confer Pikm-specific rice blast resistance. Genetics 180:2267-2276.

4. Bryan, G. T., Wu, K.-S., Farrall, L., Jia, Y., Hershey H. P., McAdams, S. A., Faulk, K. N., Donaldson, G. K., Tarchini, R., and Valent, B. 2000. A single amino acid difference distinguishes resistant and susceptible paralogs of the rice blast resistance gene Pi-ta. Plant Cell 12:2033-2046.

5. Dean, R. A., Talbot, N. J., Ebbole, D. J., Farman, M. L., Mitchell, T. K., Orbach, M. J., Thon, M., Kulkarni, R., Xu, J. R., Pan, H., Read, N. D., Lee, Y. H., Carbone, I., Brown, D., Oh, Y. Y., Donofrio, N., Jeong, J. S., Soanes, M. D., Djonovic, S., Kolomiets, E., Rehmeyer, C., Li, W., Harding, M., Kim, S., Lebrun, M. H., Bohnert, H., Coughlan, S., Butler, J., Calvo, S., Ma, L. J., Nicol, R., Purcell, S., Nusbaum, C., Galagan J. E., and Birren, B. W. 2005. The genome sequence of the rice blast fungus Magnaporthe grisea. Nature 434:980-986.

6. Don, L. D., Kusaba, M., Urashima, A. S., Tosa, Y., Nakayashiki, H., and Mayama, S. 1999. Population structure of the rice blast fungus in Japan examined by DNA fingerprinting. Ann. Phytopathol. Soc. Jpn. 65:15-24.

7. Hayashi, N. 2005. Rice Blast Fungus. MAFF Microorganism Genetic Resources Manual (18). National Institute of Agrobiological Sciences, Tsukuba, Ibaraki, Japan. (In Japanese)

8. Hirayae, K., Fujimoto, T., Yamaguchi, J., Nakajima, T., and Fujita, Y., 2008. Specific DNA markers of Pyricularia grisea isolates, Kyu9439013 and OS99-G-7a. Kyushu Plant Prot. Res. 54:1-6. (In Japanese)

9. Ishizaki, K., Matsui, T., and Harasawa, R., 2005. Practical application of multiline variety with blast resistance gene of Koshihikari in Niigata prefecture. Jpn. J. Crop. Sci. 74:438-443. (In Japanese)

10. Jia, Y., McAdams, S. A., Bryan, G. T., Hershey, H. P., and Valent, B. 2000. Direct interaction of resistance gene and avirulence gene products confers rice blast resistance. EMBO J. 19:4004-4014.

11. Kang, S., Lebrun, M. H., Farrall, L., and Valent, B. 2001. Gain of virulence caused by insertion of a Pot3 transposon in a Magnaporthe grisea avirulence gene. Mol. Plant-Microbe Interact. 14:671-674.

12. Khang, C. H., Park, S.-Y., Lee, Y.-H., Valent, B., and Kang, S. 2008. Genome organization and evolution of the AVR-Pita avirulence gene family in the Magnaporthe grisea species complex. Mol. Plant-Microbe Interact. 21:658-670.

13. Kiyosawa, S. 1984. Establishment of differential varieties for pathogenicity tests of rice blast fungus. Rice Genet. Newsl. 1:95-97.

14. Kiyosawa, S., and Cho, C. I. 1973. Relation of the sporulating ability on the rice plant to some other characters in blast fungus strains. Jpn. J. Breed. 23:239-244.

15. Koizumi, S., Iwano, M., Zenbayashi, K., dela Pena, F. A., Sonoda, R., Nakajima, T., Arai, M., Nakajima, T., Miyasaka, A., Ashizawa, T., Yasuda, N., and Noguchi, M. T. 2007. Distribution of pathogenic races of rice blast fungus in Japan in 2001. Misc. Publ. Natl. Agric. Res. Cent. 7:1-63. (In Japanese)

16. Levy, M., Romao, J., Marchetti, M. A., and Hamer, J. E. 1991. DNA fingerprinting with a dispersed repeated sequence resolves pathotype diversity in the rice blast fungus. Plant Cell 3:95-102.

17. Marchetti, M. A. 1994. Race-specific and rate-reducing resistance to rice blast in US rice cultivars. Pages 231-244 in: Rice Blast Disease. R. S. Zeigler, S. A. Leong, and P. S. Teng, eds. CAB International, Wallingford, UK.

18. Miki, S., Matsui, K., Kito, H., Otsuka, K., Ashizawa, T., Yasuda, N., Fukiya, S., Sato, J., Hirayae, K., Fujita, Y., Nakajima, T., Tomita, F., and Sone, T. 2009. Molecular cloning and characterization of the AVR-Pia locus from a Japanese field isolate of Magnaporthe oryzae. Mol. Plant Pathol. 10:361-374.

19. Nakayashiki, H., Matsuo, H., Chuma, I., Ikeda, K., Betsuyaku, S., Kusaba, M., Tosa, Y., and Mayama, S. 2001. Pyret, a Ty3/Gypsy retrotransposon in Magnaporthe grisea contains an extra domain between the nucleocapsid and protease domains. Nucleic Acids Res. 29:41064113.

20. Orbach, M. J., Farall, L., Sweigard, J. A., Chumley, F. G., and Valent, B. 2000. A telomeric avirulence gene determines efficacy for the rice blast resistance gene Pi-ta. Plant Cell 12:2019-2032.

21. Rybka, K., Miyamoto, M., Ando, I., Saito, A., and Kawasaki, S. 1997. High resolution mapping of the indica-derived rice blast resistance gene II. $P i-t a^{2}$ and $P i-t a$ and a consideration of their origin. Mol. Plant-Microbe Interact. 10:517-524.

22. Takahashi, M., Ashizawa, T., Hirayae, K., and Moriwaki, J. 2008. Estimating the mutation rate of the rice blast fungus from avirulence to virulence in the paddy field. Proc. Assoc. Plant Prot. Hokuriku 57:11-17. (In Japanese)

23. Tomita, K., Horiuchi, H., Terada, K., Kobayashi, A., Tanaka, I., Tanoi, M., Minobe, T., Furuta, H., Yamamoto, A., Masaki, N., Minami, T., Suginoto, A., Kagoshima, C., and Horiuchi, K. 2006. "Hanaechizen BL1", "Hanaechizen BL2", "Hanaechizen BL3", "Hanaechizen BL4", new rice isogenic lines with true resistant genes to blast. Bull. Fukui Agric. Exp. Stn. 43:1-16. (In Japanese)

24. Valent, B., and Chumley, F. G. 1991. Molecular genetic analysis of the rice blast fungus, Magnaporthe grisea. Annu. Rev. Phytopathol. 29:443476.

25. Yamada, M., Kiyosawa, S., Yamaguchi, T., Hirano, T., Kobayashi, T., Kushibuchi, K., and Watanabe, S. 1976. Proposal of new method for differentiating races of Pyricularia oryzae Cavara in Japan. Ann. Phytopathol. Soc. Jpn. 42:216-219.

26. Yamaguchi, T. 1980. Experimental methods for study of rice blast fungus. Pages 140-174 in: Rice Blast Disease and Breeding for Resistance. Y. Yamasaki and T. Kosaka, eds. Hakuyusha Publishers, Tokyo. (In Japanese)

27. Yoshida, K., Saitoh, H., Fujisawa, S., Kanzaki, H., Matsumura, H., Yoshida, K., Tosa, Y., Chuma, I., Takano, Y., Win, J., Kamoun, S., and Terauchi, R. 2009. Association genetics reveals three novel avirulence genes from the rice blast fungal pathogen Magnaporthe oryzae. Plant Cell 21:1573-1591.

28. Zeigler, R. S., Couc, L. X., Scott, R. P., Bernardo, M. A., Chen, D. H., Valent, B., and Nelson, R. J. 1995. The relationship between lineage and virulence in Pyricularia grisea in the Philippines. Phytopathology 85:443-451.

29. Zhou, E., Jia, Y., Singh, P., Correll, J. C., and Lee, N. F. 2007. Instability of the Magnaporthe oryzae avirulence gene AVR-Pita alters virulence. Fungal Genet. Biol. 44:1024-1034. 